\title{
Moving Towards the Age-friendly Hospital: A Paradigm Shift for the Hospital-based Care of the Elderly
}

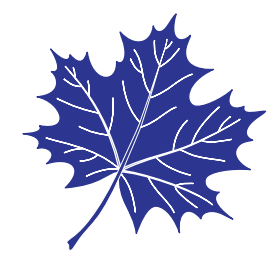

\author{
Allen R. Huang, MDCM, FRCPC, FACP, AGSF, Nadine Larente, MD, FRCPC, Jose A. Morais, MD, FRCPC \\ Division of Geriatric Medicine, McGill University Health Centre, Montreal, PQ, Canada
}

DOI:10.5770/cgj.v14i4.8

\begin{abstract}
\section{Introduction}

Care of the older adult in the acute care hospital is becoming more challenging. Patients 65 years and older account for $35 \%$ of hospital discharges and $45 \%$ of hospital days. Up to one-third of the hospitalized frail elderly loses independent functioning in one or more activities of daily living as a result of the 'hostile environment' that is present in the acute hospitals. A critical deficit of health care workers with expertise and experience in the care of the elderly also jeopardizes successful care delivery in the acute hospital setting.
\end{abstract}

\section{Methods}

We propose a paradigm shift in the culture and practice of event-driven acute hospital-based care of the elderly which we call the Age-friendly Hospital concept. Guiding principles include: a favourable physical environment; zero tolerance for ageism throughout the organization; an integrated process to develop comprehensive services using the geriatric approach; assistance with appropriateness decision-making and fostering links between the hospital and the community. Our current proposed strategy is to focus on delirium management as a hospital-wide condition that both requires and highlights the Geriatric Medicine specialist as an expert of content, for program development and of evaluation.

\section{Conclusion}

The Age-friendly Hospital concept we propose may lead the way to enable hospitals in the fast-moving health care system to deliver high-quality care without jeopardizing risk-benefit, function, and quality of life balances for the frail elderly. Recruitment and retention of skilled health care professionals would benefit from this positive 'branding' of an institution. Convincing hospital management and managing change are significant challenges, especially with competing priorities in a fiscal environment with limited funding. The implementation of a hospital-wide delirium management program is an example of an intervention that embodies many of the principles in the Age-friendly Hospital concept. It is important to change the way hospital care is delivered to older adults in time to meet our needs when we need hospital services ourselves.

Keywords: age-friendly, hospital services, adverse outcomes, models of care

\section{INTRODUCTION}

A "perfect storm" is brewing in health care for older adults. The conditions include an increasing number of elderly patients requiring and presenting for health services in the acute care hospitals, a hospital environment that is ill-suited to the needs of those patients, and a serious and sustained lack of health professionals with special training or expertise in the care of the elderly. We reviewed the current literature with the focus on new models of care for older patients in our acute care hospitals. We propose a concept, which we call the 'Age-friendly Hospital' (AFH), in order to change the way we do things to enable patients to reap the benefits of advanced medical and technological care, as well as to avoid excess morbidity and mortality in an environment and involving processes that are currently not well-suited for older adults.

\section{The Demographic Imperative}

The successes of improved secular hygiene measures and modern health care interventions have resulted in everincreasing numbers of people living longer. We are currently witnessing, and will continue to witness over the next 30 years, a sharp increase in the number of persons over 65 years of age. In late 2011, the world population reached 7 billion. As estimated by the World Health Organization in 2009, based on the reports of its 193 member states, $11 \%$ of this population was older than 60 years. ${ }^{(1)}$ More specific examples show that in 2008 the percent of people 65 years 
and older was approximately $14 \%$ in the US, $16 \%$ in the United Kingdom, $15 \%$ in Canada, 20\% in Germany, and $22 \%$ in Japan. ${ }^{(2)}$ The fastest growing segment of the population are those aged 85 years and over. This age group is recognized for carrying the greatest disease burden and for suffering the most from functional impairment. People 65 years and older accounted for approximately $60 \%$ of health care expenditures, $35 \%$ of hospital discharges, and $45 \%$ of hospital days. ${ }^{(3)}$

\section{The Acute Care Hospital}

Seniors admitted to acute hospitals are more likely to have multiple chronic diseases, as well as impaired cognition and higher levels of dependency (including mobility) than younger adults. These patients stay longer in hospital, have a greater risk for functional decline, and require nursing home placement. Acute hospitals frequently present a "hostile environment" that leads to functional decline in the frail elderly and a "cascade of dependency" that results in approximately one-third of older patients losing independent functioning in one or more activities of daily living. ${ }^{(4)}$ This decline is not related to acute illnesses but to the adverse effects of modern therapy and current hospital practices, which are designed for younger people.

\section{Lack of Health Care Workers with Specialized Knowledge}

There is a severe and sustained shortage of healthcare workers with specialized training and experience in Geriatrics. The Institute of Medicine 2008 report, Retooling for an Aging America, describes the scope of the problem. ${ }^{(5)}$ For example, the report estimates that 36,000 geriatricians and 3.5 million formal healthcare providers will be needed by the year 2030 to meet the needs of the US population. A multipronged approach was suggested, including enhancing the competence of all care providers, as well as expanding the training of healthcare workers.

\section{Literature Review}

A literature review was conducted using the OvidSP search engine (Wolters Kluwer, Alphen aan den Rijn, The Netherlands) to access the MEDLINE and Embase electronic bibliographic databases. We searched the English literature from January 1996 to February 2011 using the following terms in combination: aged, elderly and aging with acute care hospital and age or elder-friendly hospital. A web search using the Google search engine was also done to find relevant web pages. Our hospital division of Geriatric Medicine also held a strategic planning exercise to respond to the issue of senior management. It questioned the ongoing role and presence of our specialty in the acute care academic hospitals.

\section{DISCUSSION}

To provide context, Montreal is the largest city in the province of Quebec, with a metropolitan population of over two million. The McGill University Health Centre (MUHC) is the main academic health institution for McGill University and is spread across the merged institutions of the Montreal General Hospital, Royal Victoria Hospital, Montreal Children's Hospital, Montreal Neurological Institute, the Montreal Chest Institute, and the Lachine General Hospital. The MUHC operates on average 880 adult and 120 pediatric beds, with a volume of 36,000 admissions and 600,000 ambulatory visits per year. Our Geriatric Medicine service has 38 inpatient beds on two distinct units. Our annual volumes involve approximately 400 inpatient admissions and 1,400 consultations in the Emergency Department. We have seven full-time equivalent physicians who provide these services.

There were a total of 11 articles and 14 webpages considered in the detailed literature review. The majority of articles are nursing practice-based. Analogous to the concept of children-friendly hospitals and in response to the World Health Organization initiative of Age-friendly Environments program $^{(6)}$ and age-friendly communities, ${ }^{(7)}$ the concept of the 'Elderly-friendly Hospital' has been proposed by nursing management initially by Parke and Stevenson in $1999^{(8)}$ and by Parke and Brand in 2004. ${ }^{(9)}$ Subsequently in 2009, Chiou and Chen ${ }^{(10)}$ proposed a framework to promote the health, dignity, and participation of older hospitalized adults. After our review and analyses, we proposed a specific set of guiding principles. Rather than compartmentalizing the role of specialized geriatric services in order to respond to the challenges of managing only a portion of the hospitalized frail elderly, we propose that the guiding principles listed below be applied to the institution as a whole, so that all older patients, family members, and visitors can benefit. These principles, along with examples of their implementation, are as follows:

1. A favourable physical environment.

a. Integrate and implement designs that are age-friendly and respond to the needs of patients, families and visitors with a variety of impairments (physical mobility, endurance, visual, hearing, cognitive)

b. Introduce innovative designs that promote the maintenance of function and independence in the hospitalized frail elderly ${ }^{(11,12)}$

2. Zero tolerance towards ageism at all levels of the organization.

a. The "Age-friendly" vision has to be fully endorsed as a strategic objective by the institutional administration

b. Knowledge, skills and attitudes of all care providers are supportive of this objective, along with training opportunities to meet it ${ }^{(5)}$

c. A marketing approach, such as linking this concept to the slogan "Age-aware means better care", can 
potentially enable healthcare worker recruitment and retention, as well as be attractive to patients and their families

3. An integrated process to develop comprehensive services using principles of the geriatric approach across the entire institution by implementing:

a. A geriatric interdisciplinary consult team in the Emergency Department to help with the early identification of high-risk patients, following the concepts promoted by McCusker et al. ${ }^{(13,14)}$

b. A systematized program such as the Hospital Elder Life Program (HELP) ${ }^{(15)}$ to prevent delirium and functional decline for intermediate and high-risk patients

c. A specialized unit for the management of patients with behaviour manifestations associated with dementia and delirium

d. An Acute Care for Elders (ACE) unit for patients above a targeted age (e.g., 85 years $)^{(16,17)}$

e. Co-management by Geriatrics of patients within the services of orthopaedic surgery, vascular surgery, cardiac surgery, and cancer care

f. A comprehensive nutrition support program, from food preparation to delivery, presentation, and including feeding assistance

g. An elder-assist program to help patients remember their appointments, navigate the hospital services, understand the outcomes of health care discussions, and advocate for their choices

4. Assistance with appropriateness decision-making (e.g., Levels of care and Interventions: Too much vs. Not enough).

a. Interdisciplinary service to assess older in-patients and out-patients who are being considered for advanced surgeries and interventions. Interventions should be patient-centered and have measureable benefits

5. Fostering links between the acute care hospital and the community.

a. The right patient, cared for in the right place, at the right moment.

b. Promote existing ties within regional health care jurisdictions to enable clinical excellence, teaching, and research opportunities

c. Innovative programs of subacute and postacute care of the frail elderly and patients with multiple chronic conditions (rehab institutions, skilled nursing homes, other)

The aims of developing an age-friendly hospital are to promote excellence in hospital care for acutely ill older adults through the provision of evidenced-based service delivery and patient-family focused-care, and to ensure that the "geriatric approach" is incorporated into practice standards across all patient care programs and services within the hospital. While we are cognizant of the increase in human resources and associated costs needed to effectively implement an AFH program, we anticipate savings generated through a reduction in hospitalization-related complications, avoidance of inappropriate tests and procedures, decreased visits to the Emergency Department, and possibly decreased healthcare worker burnout. Programs such as the Hospital Elder Life Program (HELP) have already demonstrated a positive cost-benefit. ${ }^{(15)}$ Although the AFH program may not be initially cost-neutral, the recommendation at present consists in evaluating all components of the new proposed changes in care, including the perceived quality of care by our target population and their family members. Patient-reported outcomes involving accessibility and acceptability have been foundational concepts for hospital accreditation activities, such as those promoted by Accreditation Canada (www.accreditation.ca) and should be seriously considered. An additional advantage of the AFH concept would be the positive 'branding' bestowed upon an institution, which could help recruitment and retention of skilled healthcare professionals.

\section{Challenges and Potential Opportunities}

One significant challenge is convincing hospital senior management of the value of geriatric services. Our personal adventures began after a gloomy discussion with hospital senior management during the Fall of 2008 when we were informed that Geriatric services were going to soon be designated as optional within the inpatient services setup. Our division responded by submitting a white paper outlining the guiding principles for the development of an elderly-friendly hospital. To our surprise, in January 2009, the same senior management and hospital redevelopment and planning committees accepted the principles outlined in our white paper. Serendipity also contributed to the start of an organization-wide clinical activities priority-setting exercise. After the reports from 92 hospital services were analyzed, Geriatric Medicine was placed at a medium priority and the age-friendly hospital principles were proposed as one of the cross-cutting pillars for the future.

Another significant challenge is fiscal. Administrative data is a strong potential ally. Drilling down into detailed patient tracking data has shown that when our Geriatrics unit is compared to a general Internal Medicine unit, the in-patient acute length-of-stay is predictably longer (28.7 d vs. $15.1 \mathrm{~d}$ ), but patients discharged home were one-third as likely to return to the Emergency Department within 14 days of discharge (5.3\% vs. $17 \%)$ and require re-admission ( $3 \%$ vs. $10 \%$ ). A more detailed cost analysis of longitudinal health services utilization rather than crude episodic numbers would need to be done in order to determine the value proposition of investments required and operational 
sustainability of implementing some of the principles of an age-friendly hospital. Communication of ideas and concepts to patient support groups and government may also be fruitful. Our efforts captured the interest of the Quebec Minister for Families and the Elderly (Ministère de la famille et des aînés) who recently invested the amount of $\$ 300,000$ as a grant to initiate our pilot project.

\section{CONCLUSION}

The concept of the age-friendly hospital is promising in terms of fulfilling the care needs of older hospitalized adults who make up the largest portion of clientele in the acute care system, while ensuring a high quality of care at the best cost-benefit ratio. We hope that the AFH concept will stimulate further discussion about models of care for the elderly in the acute hospital environment, as well as research into the effectiveness of these models. We think that the positive 'branding' of the AFH concept endows an institution to improve recruitment and retention of skilled health care professionals. The funding challenges are not insurmountable, since reallocation of existing ill-spent funds or lobbying for new investments can help initiate pilot projects that can establish momentum. We wish to change the way hospital care is delivered to older adults, in time to meet our needs when we need them ourselves.

\section{ACKNOWLEDGEMENTS}

This research was done without specific funding support. None of the authors received special funding. This research has previously been reported as an abstract and a poster at the 19th International Association of Gerontology and Geriatrics World Congress, Paris, France, July 5-9, 2009 and at the 29th Annual Scientific meeting, Canadian Geriatrics Society, Toronto, Ontario, April 23-25, 2009.

\section{CONFLICT OF INTEREST DISCLOSURES}

The authors declare that they have no competing interests.

\section{REFERENCES}

1. World Health Organization. World Health Statistics 2011. Geneva, Switzerland: WHO Press; 2011.

2. Percentage of population age 65 or over. Available from: http://www.data360.org/dsg.aspx?Data_Set_Group_Id=466. Accessed November 28, 2011.

3. The aging of America. Available from: http://www.prcdc. org/300million/The_Aging_of_America/. Accessed November $28,2011$.
4. Creditor MC. Hazards of hospitalization of the elderly. Ann Intern Med. 1993;118(3):219-223.

5. Institute of Medicine. Retooling for an aging America: building the healthcare workforce. Report of the Institute of Medicine Committee on the Future of Health Care Workforce for Older Americans. Washington, DC: The National Academies Press; 2008.

6. World Health Organization. WHO age-friendly environments programme. Available from: http://www.who.int/ageing/ age_friendly_cities/en/index.html. Accessed November 28, 2011.

7. Lui C-W, Everingham J-A, Warburton J, et al. What makes a community age-friendly: a review of international literature. Australa J Ageing. 2009;28(3):116-121.

8. Parke B, Stevenson L. Creating an elder-friendly hospital. Healthcare Management Forum. 1999;12:45-48.

9. Parke B, Brand P. An elderly-friendly hospital: translating a dream into reality. Nurs Leadersh. 2004;17(1):62-77.

10. Chiou S-T, Chen L-K. Towards age-friendly hospitals and health services. Arch Gerontol Geriat. 2009;49(Suppl 2):S3-S6.

11. McCarthy M. Healthy design. Lancet. 2004;364:405-406.

12. Fraser Health Authority/Simon Fraser University Research Project. Towards more elder friendly acute hospitals: a program of research focused on the physical environment of hospitals in the Fraser Health Authority. Available from: www.sfu.ca/ livinglab/project_gutman_EF_ACU.doc.

13. McCusker J, Bellavance F, Cardin S, et al. Detection of older people at increased risk of adverse health outcomes after an emergency visit: the ISAR screening tool. $J$ Am Geriatr Soc. 1999;47(10):1229-1237.

14. McCusker J, Verdon J, Tousignant P, et al. Rapid emergency department intervention for elders reduces risk of functional decline: results of a multi-centre randomized trial. J Am Geriatr Soc. 2001;49(10):1272-1281.

15. Inouye SK, Bogardus ST, Baker D, et al. The hospital elder life program: a model of care to prevent cognitive and functional decline in older hospitalized patients. J Am Geriatr Soc. 2000;48(12):1697-1706.

16. Landefeld CS, Palmer RM, Kresevic DM, et al. A randomized trial of care in a hospital medical unit especially designed to improve the functional outcomes of acutely ill older patients. N Engl J Med. 1995;332:1338-1344

17. Palmer RM, Counsell SR, Landefeld SC. Acute care for elders units: practical considerations for optimizing health outcomes. Dis Manag Health Out. 2003;11(8):507-517.

Correspondence to: Allen R. Huang, MDCM, FRCPC, FACP, AGSF, Division of Geriatric Medicine, McGill University Health Centre, Royal Victoria Hospital, 687 Pine Avenue West, Room M8.12, Montréal, Quebec, Canada H3A 1A1

E-mail: allen.huang@muhc.mcgill.ca 